\title{
Diffraction at HERA
}

\author{
P. Marage \\ Université Libre de Bruxelles, CP 230 - B-1050 Brussels - Belgium - pmarage@ulb.ac.be
}

\begin{abstract}
Recent results on inclusive and exclusive diffraction at HERA are presented.
Keywords: HERA, diffraction, structure functions, jets, charm, DVCS

PACS: $13.60 .-\mathrm{r}, 13.60 . \mathrm{Hb}, 13.85 . \mathrm{Hd}, 13.85 . \mathrm{Ni}$
\end{abstract}

Diffractive processes, characteristic of strong interactions, are intimately related to elastic scattering. When the quantum states which build up the interacting particles undergo elastic scatterings with differing amplitudes, their subsequent rearrangement leads to the excitation of one or both of the initial particles into higher mass systems [1]. At high energy, the process is parameterised through the exchange of the pomeron $I P$, an object carrying the vacuum quantum numbers.

At the HERA $e-p$ collider, diffractive photon-proton interactions contribute $\simeq 10 \%$ of the total deep-inelastic scattering (DIS) cross section (i.e. for photon virtualities $Q^{2}>$ a few $\mathrm{GeV}^{2}$ ). They are characterised by the presence of two systems separated by a large gap in rapidity: a hadronic system $X$, with mass $M_{X}$, which carries the photon quantum numbers, and an elastically scattered or diffractively dissociated proton (system $Y$, with mass $M_{Y}$ ), emitted at small angle and which carries most of the initial proton momentum.

Diffractive $\gamma-p$ interactions are due to the differential scattering amplitudes of the various components of the photon wave. At lowest orders in QCD, these components are different size colour dipoles ( $q-\bar{q}$ and $q \bar{q}-g$ photon fluctuations), and the exchange is mediated by two gluons. In contrast to soft interactions which imply large size dipoles, small dipole scattering, selected by the presence of a hard scale, allows QCD perturbative calculations.

The hard scale can be provided by the photon virtuality $Q^{2}$, a heavy quark mass ( $c$ or $b$ production), large transverse momentum jet production, or a large value of $t$, the fourmomentum transfer squared to the proton (or system $Y$ ). Because of colour screening, small size dipoles have small cross sections.

In a complementary approach, a factorisation theorem justifies the definition in the DIS domain of diffractive structure functions, which follow QCD evolution [2]. They depend on four dynamical variables, for fixed $M_{Y}: Q^{2}$, the Bjorken variable $x$, the momentum fraction lost by the scattered proton $\xi$, and $t$. Universal diffractive parton distribution functions (pdf's) can thus be defined, which govern exclusive processes.

A further simplifying assumption, inspired by Regge phenomenology, is proton vertex factorisation, i.e. the factorisation of diffractive pdf's into a pomeron flux in the proton (depending on $x_{I P}=1-\xi$ and $t$ ), and pomeron pdf's (depending on $Q^{2}$ and $\beta=x / x_{I P}$, similar to the real hadron case). Is this framework, $x_{I P}(\beta)$ is the momentum fraction of the proton (pomeron) carried by the pomeron (struck quark). 
The fully differential structure function $F_{2}^{D(4)}\left(Q^{2}, \beta, x_{I P}, t\right)$ has been measured by the $\mathrm{H} 1$ and ZEUS collaborations, using proton spectrometers [3, 4]. A recent $\mathrm{H} 1$ measurement [4] confirms the semi-hard nature of inclusive diffraction (the slope of the exponentially falling $t$ distribution $\simeq 6 \mathrm{GeV}^{-2}$ ), and supports vertex factorisation.
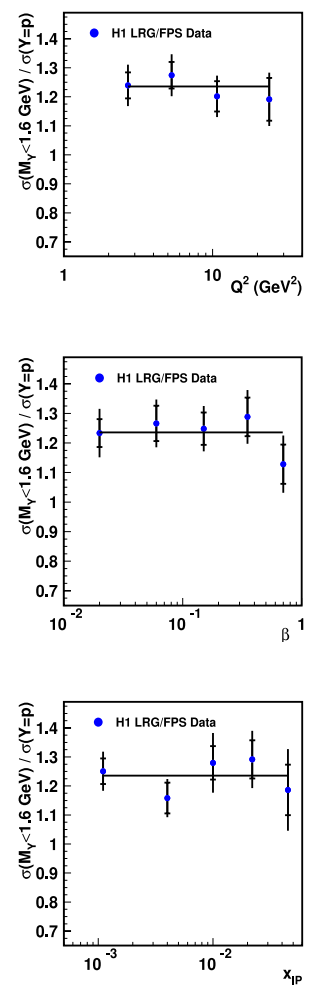
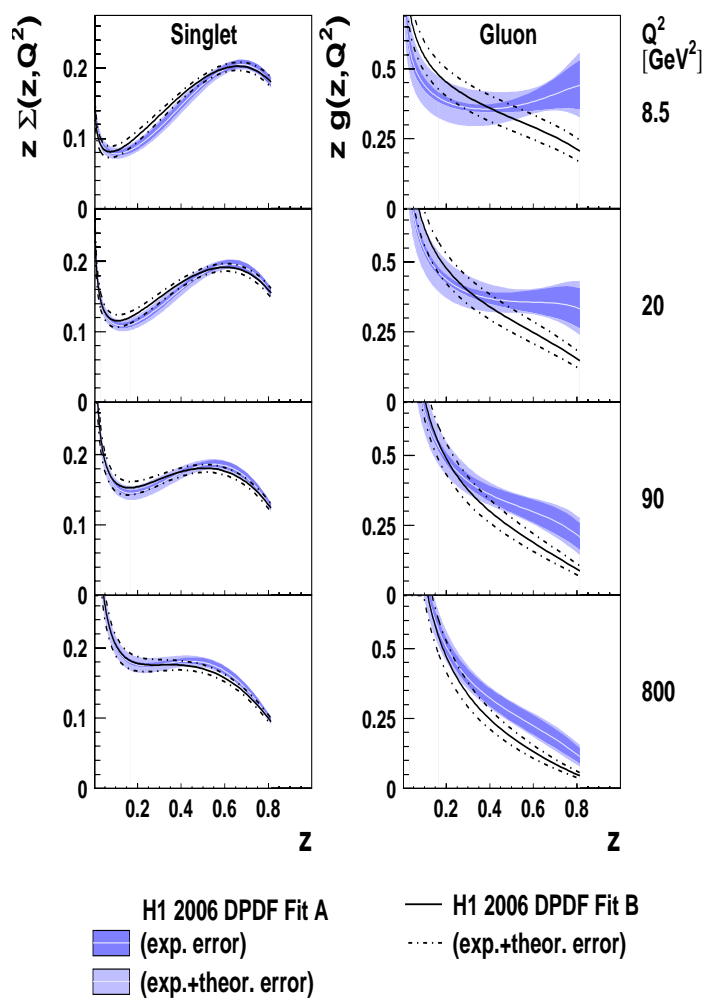

- H1 2006 DPDF Fit B

$\cdots$ (exp.+theor. error)

FIGURE 1. Left: $Q^{2}, \beta$ and $x_{I P}$ independence of the diffractive cross section ratio $\sigma\left(m_{p} \leq M_{Y} \leq\right.$ $1.6 \mathrm{GeV}) / \sigma(Y=p)$ [4]. Right: quark (left) and gluon (right) pdf central values and uncertainties, for several $Q^{2}$ values; the two fits differ in the starting parameterisations of the QCD evolution [7].

The $F_{2}^{D(3)}\left(Q^{2}, \beta, x_{I P}\right)$ structure function, integrated over $t$, has been measured by $\mathrm{H} 1$ and ZEUS, selecting events with a large rapidity gap or - correspondingly - with a nonexponentially suppressed large $M_{X}$ mass $[5,6]$. Scaling violations up to large $\beta$ values ( $\beta \simeq 0.6$ ) indicate that the diffractive exchange is dominated by gluons.

In the extraction of diffractive pdf's [5,3], the quark distribution is rather precisely measured, but large systematic uncertainties still affect the gluon distribution, which is only indirectly measured through the QCD evolution. From the most recent H1 measurements [7], two gluon parameterisations which differ mainly at large $\beta$ values are extracted (see Fig. 1, right), with similar $\chi^{2} / d o f$.

The universality of diffractive pdf's has been tested in processes implying different hard scales, i.e. heavy quark and transverse jet photo- and electroproduction (LO and NLO calculations), which are sensitive to the diffractive gluon content of the proton through photon-gluon-fusion amplitudes. A qualitative agreement with the expectations is observed for charm production (Fig. 2, left); however large errors arise from charm quark mass and scale uncertainties (shaded band), and from limited statistics.

For diffractive jet production, an agreement is observed in the DIS case, although 

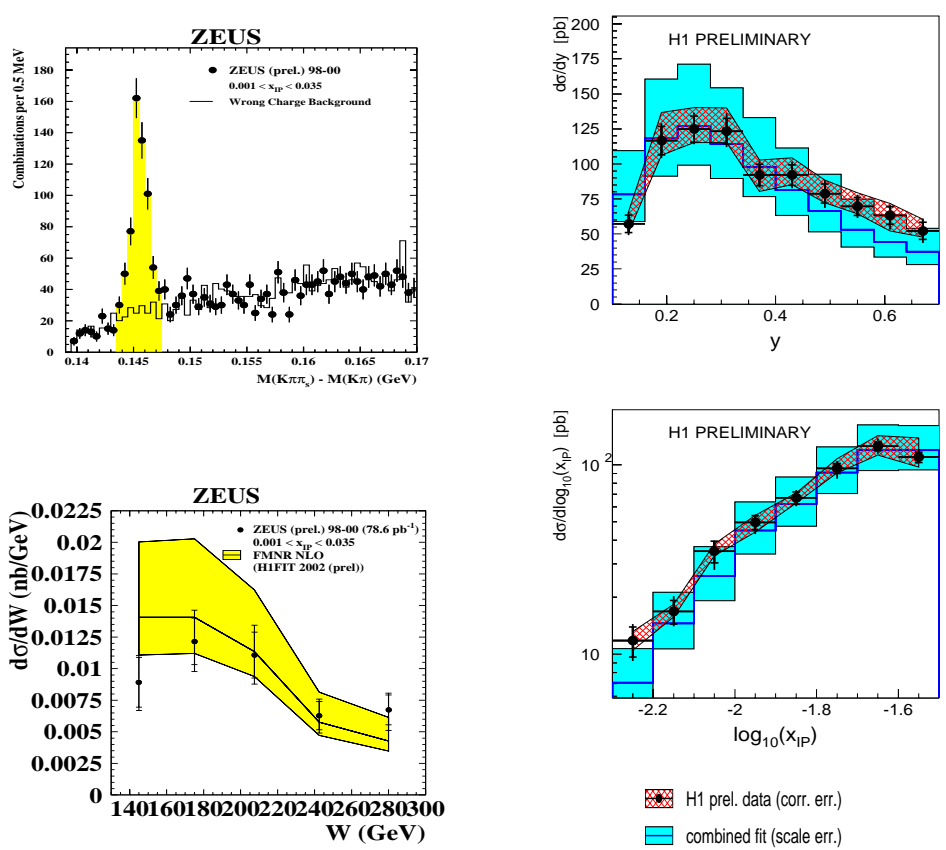

FIGURE 2. Left: diffractive charm photoproduction signal (top), and comparison with NLO predictions using diffractive pdf's (bottom) [8]. Right: $y\left(y=Q^{2} / x s\right.$, with $s=e-p$ invariant mass) (top) and $x_{I P}$ (bottom) distributions of diffractive DIS jet production, using pdf's extracted from a joint fit to diffractive inclusive DIS and jet production [9].

the predictions are affected by the large systematic errors on the gluon pdf. Joint fits to diffractive inclusive DIS and jet production measurements result in values of $\chi^{2} / d o f$ comparable to those for fits to inclusive data alone, and can thus provide further constraints and increased precision to the gluon distribution. Resulting predictions for DIS jets are compared to data on Fig. 2, right; the band shows the scale uncertainty. In photoproduction, in contrast, indications are found for factorisation breaking $[10,11]$; this is attributed to rescattering between the proton and parton remnants of resolved photons, but surprisingly it is also observed in the kinematic domain where direct photons are expected to dominate $\left(x_{\gamma} \simeq 1\right)$.

Exclusive vector particle photo- and electroproduction have been extensively studied at HERA: deeply virtual Compton scattering (DVCS), $\rho, \omega, \phi, J / \Psi$ and $\Upsilon$ production.

For the DVCS process - which may give access to Generalised Parton Distributions -, the energy dependence measured by $\mathrm{H} 1$ and ZEUS $[12,13]$ for $Q^{2}>$ a few $\mathrm{GeV}^{2}$ is typical of hard processes, like $J / \Psi$ or high $Q^{2} \rho$ production (see Fig. 3, top left); however the measured $t$ dependence [12] is softer than for $J / \Psi$ production (Fig. 3, bottom left).

High $t$ vector meson production $(\rho, J / \Psi)$ provides tests of QCD BFKL calculations. In $\rho$ photoproduction, H1 has recently [14] confirmed the ZEUS result [15] of a large double spin-flip amplitude (see Fig. 3, right). This is in qualitative - but not really quantitative - agreement with BFKL calculations.

In conclusion, HERA collider results have led over the past few years to major progress in the detailed QCD understanding of diffraction. Precision measurements are performed of the diffractive structure functions, and parton distribution functions 

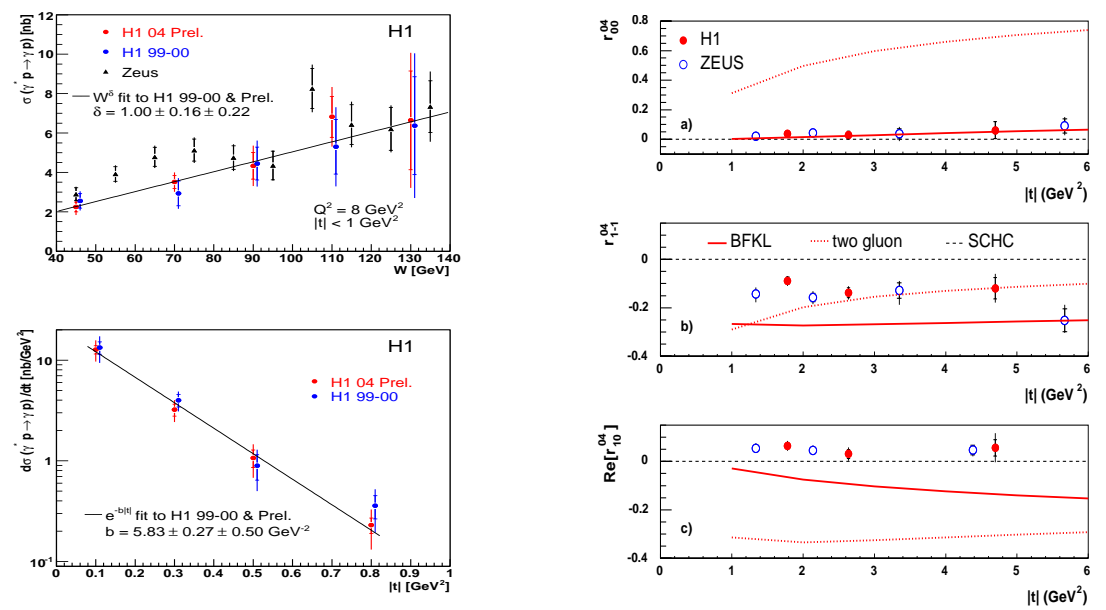

FIGURE 3. Left: $W$ and $t$ dependences of DVCS production ( $W$ is the $\gamma-p$ hadronic system invariant mass) [12]. Right: $t$ dependence of spin density matrix elements for $\rho$ meson photoproduction, compared to BFKL predictions (full lines) [14].

are extracted, although with still rather large errors for gluons at large $\beta$. Significant statistics of diffractive events in the presence of a hard scale (jets, charm production) allow tests of diffractive factorisation, and joint fits with inclusive diffractive DIS lead to improved pdf determination. Among numerous diffractive studies, vector meson production and DVCS provide additional insight into QCD processes, in particular BFKL evolution.

A better understanding of diffractive factorisation and of underlying factorisation breaking effects ("survival probability") are of major importance for LHC, in particular for attempts at discovering diffractive Higgs production.

\section{REFERENCES}

1. M.L. Good and W.D. Walker, Phys. Rev. 120 (1960), 1857.

2. J.C. Collins, Phys. Rev. D 57 (1998), 3051; 61 (2000), 019902.

3. S. Chekanov et al., ZEUS Coll., Eur. Phys. J. C 38 (2004), 43.

4. A. Aktas et al., H1 Coll., DESY 06-048, subm. to Eur. Phys. J. C.

5. C. Adloff et al., H1 Coll., Z. Phys. C 76 (1997), 613.

6. S. Chekanov et al., ZEUS Coll., Nucl. Phys. B 713 (2005), 3.

7. A. Aktas et al., H1 Coll., DESY 06-049, subm. to Eur. Phys. J. C.

8. ZEUS Coll., Diffractive photoproduction of $D^{* \pm}(2010)$ mesons at HERA, ZEUS-prelim-05-022-plus, subm. to ICHEP06.

9. H1 Coll., Diffrative parton densities from a combined analysis of dijets and inclusive data in diffractive DIS, H1-prelim-06-016, subm. to DIS2006.

10. ZEUS Coll., Diffractive photoproduction of dijets at HERA, ZEUS-prel-04-011, subm. to ICHEP04.

11. H1 Coll., Dijets in Diffractive Photoproduction and Deep-Inelastic Scattering at HERA, H1prelim04-113, subm. to ICHEP04.

12. H1 Coll., DVCS with HERA II data, H1-prelim-06-012, subm. toDIS2006.

13. S. Chekanov et al., ZEUS Coll., Phys. Lett. B 573 (2003), 46.

14. A. Aktas et al., H1 Coll., DESY 06-023, subm. to Phys. Lett. B.

15. S. Chekanov et al., ZEUS Coll., Eur. Phys. J. C 26 (2003), 389. 